\title{
Hypofractionated radiotherapy for macroscopic canine soft tissue sarcoma: A retrospective study of 50 cas-es treated with a $5 \times 6$ gy protocol with or without metronomic chemotherapy
}

\author{
Cancedda, Simona ; Marconato, Laura ; Meier, Valeria Sabina ; Laganga, Paola ; Roos, Malgorzata ;
} Leone, Vito F ; Rossi, Federica ; Rohrer Bley, Carla

\begin{abstract}
Wide surgical resection or a marginal/incomplete resection followed by full-course radiation therapy is the current standard of care for canine soft tissue sarcoma. The purpose of this retrospective, descriptive, bi-institutional study was to determine the effectiveness and toxicity of a hypofractionated $5 \times 6$ Gy protocol on macroscopic canine soft tissue sarcoma in terms of progression-free interval (PFI) and overall survival (OS), and to identify prognostic factors for patient outcome. Dogs with macroscopic soft tissue sarcoma irradiated with $5 \times 6$ Gy were eligible for the study. Progression-free interval and OS were compared with respect to different tumor and patient characteristics by the Kaplan-Meier method and multivariable Cox regression analysis. Fifty dogs with macroscopic disease were included. All dogs received the same radiation therapy protocol; part of the group $(\mathrm{n}=20)$ received postradiation metronomic chemotherapy. Median PFI for all cases was 419 days (95\% confidence interval (CI): 287551 ) and median OS was 513 days (95\% CI: 368-658). Dogs with tumors on the limbs had significantly longer PFI and OS, compared with head or trunk. Increasing tumor burden decreased OS. The addition of metronomic chemotherapy yielded a significantly longer OS (757 days (95\% CI: 570-944) compared with dogs that did not receive systemic treatment (286 days (95\% CI: $0-518),(\mathrm{P}=0.023)$ ), but did not influence progression-free interval. Toxicity was low throughout all treatments. The $5 \times 6$ Gy radiation therapy protocol was well tolerated and provided long PFI and OS in dogs with macroscopic soft tissue sarcoma.
\end{abstract}

DOI: https://doi.org/10.1111/vru.12308

Posted at the Zurich Open Repository and Archive, University of Zurich

ZORA URL: https://doi.org/10.5167/uzh-116570

Journal Article

Accepted Version

Originally published at:

Cancedda, Simona; Marconato, Laura; Meier, Valeria Sabina; Laganga, Paola; Roos, Malgorzata; Leone, Vito F; Rossi, Federica; Rohrer Bley, Carla (2016). Hypofractionated radiotherapy for macroscopic canine soft tissue sarcoma: A retrospective study of 50 cas-es treated with a $5 x 6$ gy protocol with or without metronomic chemotherapy. Veterinary Radiology Ultrasound, 57(1):75-83.

DOI: https://doi.org/10.1111/vru.12308 
1 Hypofractionated Radiotherapy for Macroscopic Canine Soft Tissue Sarcoma: A

2 Retrospective Study of 50 Cases Treated with a 5x6 Gy Protocol with or without Metronomic

3 Chemotherapy

4

5 Simona Cancedda $^{1 *}$, Laura Marconato ${ }^{1}$, Valeria Meier ${ }^{2}$, Paola Laganga ${ }^{1}$, Malgorzata Roos ${ }^{3}$,

6 Vito F. Leone ${ }^{1}$, Federica Rossi ${ }^{1}$, Carla Rohrer Bley ${ }^{2 *}$

7

$8{ }^{1}$ Centro Oncologico Veterinario, 40037 Sasso Marconi (BO), Italy

$9 \quad{ }^{2}$ Division of Radiation Oncology, Vetsuisse-Faculty, University of Zurich, CH-8057

10 Zurich, Switzerland

$11{ }^{3}$ Division of Biostatistics, Epidemiology Biostatistics and Prevention Institute, Faculty of

12 Medicine, University of Zurich, CH-8001 Zürich, Switzerland

13

14

15

*equally contributing authors

16

17 Running head: Radiotherapy for Macroscopic Canine Soft Tissue Sarcoma

18 Key words: radiotherapy, dog, soft tissue sarcoma, metronomic chemotherapy

20 Corresponding author: C. Rohrer Bley, Division of Radiation Oncology, Vetsuisse-Faculty,

21 University of Zurich, Winterthurerstrasse 260; CH-8057 Zurich, Switzerland

22 Tel: +41 44635 8487; Fax: +41 44635 8940; E-mail: crohrer@vetclinics.uzh.ch

23

24 Funding sources: None

25 Previous presentations or abstracts: ESVONC 2014, Vienna, Austria 
Wide surgical resection or a marginal/incomplete resection followed by full-course radiation therapy is the current standard of care for canine soft tissue sarcoma. The herein presented results of a retrospective, cross-sectional, bi-institutional study describe the effectiveness and toxicity of a hypofractionated 5x6 Gy protocol on macroscopic canine soft tissue sarcoma in terms of progression-free interval and overall survival, identifying prognostic factors on outcome.

Dogs with macroscopic soft tissue sarcoma irradiated with 5x6 Gy were eligible for the study.

34 Progression-free interval and overall survival were compared with respect to different tumor and patient characteristics by the Kaplan-Meier-method and multivariable Cox-regression analysis. Fifty dogs with macroscopic disease were included. All dogs received the same radiation therapy protocol; part of the group $(n=20)$ received post-radiation metronomic chemotherapy. Median progression-free interval for all cases was 419 days (95\%CI:287-551) and median overall survival was 513 days (95\%CI:368-658). Dogs with tumors on the limbs had significantly longer progression-free interval and overall survival, compared with head or trunk. Increasing tumor burden decreased overall survival. The addition of metronomic chemotherapy yielded a significantly longer overall survival (757 days (95\%CI:570-944) compared with dogs that did not receive systemic treatment (286 days (95\%CI:0-518), $(p=0.023))$, but did not influence progression-free interval. Toxicity was low throughout all treatments. The 5x6 Gy radiation therapy protocol was well tolerated and provided long progression-free interval and overall survival in dogs with macroscopic soft tissue sarcoma. 


\section{Introduction}

Soft tissue sarcomas refer to a group of malignant tumors that arise from mesenchymal cells and can occur in any part of the body, most commonly in the extremities and trunk. Although canine soft tissue sarcomas present with wide variations in subtypes (fibrosarcoma, perivascular wall tumors, peripheral nerve sheet tumors, liposarcoma, myxosarcoma, malignant mesenchymoma $)^{1,2}$, these tumors tend to have a similar biologic behavior, consisting of locally invasive growth and usually a low-to-intermediate metastatic potential. Conversely, other mesenchymal tumors also arising from soft tissues, including hemangiosarcoma, histiocytic sarcoma and rhabdomyosarcoma, have a more aggressive biologic behavior and a higher tendency to spread. ${ }^{3}$ Surgery with or without full-course postoperative radiation therapy is the mainstay of treatment of canine soft tissue sarcomas. ${ }^{2,4-6}$ Adequate treatment yields long survival times ranging from $1416^{3}$ to 2270 days ${ }^{4,5}$, with only $33 \%$ of the cases succumbing to the disease. However, analyses of prognostic factors showed that the extent of resection, initial tumor size, anatomical location, histological grade and clinical stage were predictive of outcome..$^{3-5}$ It is well documented that the extent of resection is associated with local control of soft tissue sarcoma. ${ }^{6,7}$ However, one study describes that some of these tumors resected without an adequate margin of normal tissue may only have a failure rate of about $30 \% .{ }^{8}$ Unfortunately, in some clinical situations, surgery cannot be used without unacceptable morbidity, or gross disease is left behind at resection in an attempt to spare critical normal structures or avoid amputation in the case of soft tissue sarcoma located on the extremities.

Hypofractionated radiation therapy protocols for macroscopic or microscopic canine soft tissue sarcomas are currently used in a true palliative (e.g. providing pain relief and improvement of dysfunction $)^{9,10}$ setting for elderly patients or patients presenting with serious co-morbidities, but also due to financial restraint of owners or logistical restraint of treatment 
72 machine availability. ${ }^{11-13}$ The responses in the macroscopic setting using a 4x8 Gy weekly

73 protocol have been found to be rather short-lived with about 5 months of median time to

74 progression. ${ }^{11}$ Traditional dose-intense chemotherapy as a single treatment modality has not

75 proven to be effective in local tumor control; nevertheless, the administration of metronomic

76 chemotherapy has recently shown to delay tumor recurrence after incomplete resection of

77 canine soft tissue sarcomas ${ }^{14}$, most likely by inhibiting tumor angiogenesis and suppressing

78 regulatory T-cells. ${ }^{15}$

79 With these considerations in mind, this retrospective, cross-sectional, bi-institutional study

80 was conducted to assess the effectiveness of a previously published 5x6 Gy hypofractionated

81 radiation therapy protocol ${ }^{13,16-19}$, with or without post-radiation metronomic chemotherapy, in

82 dogs with macroscopic (including unresected or recurrent) soft tissue sarcoma, and to

83 evaluate tumor and treatment variables associated with local control and survival. It was

84 hypothesized that the hypofractionated radiation therapy protocol would result in good

85 tolerability and provide long progression-free interval and overall survival. 
Medical records of dogs with soft tissue sarcomas admitted to the Division of Radiation

Veterinario, Sasso Marconi, Bologna, between 2006 and 2013 were reviewed. Due to the retrospective nature of this study, reporters were aware of outcome at the time of data recording.

Dogs were included in the study if they had a soft tissue sarcoma that was diagnosed by means of histopathology, underwent a 5x6 Gy radiation therapy protocol in a macroscopic setting with or without post-radiation metronomic chemotherapy. Dogs were excluded if their tumor occurred in the oral or nasal cavity, if they had received previous chemotherapy and/or radiation therapy, or in the case of histopathological diagnosis demonstrating histiocytic sarcoma, angiosarcoma or rhabdomyosarcoma, due to the documented different biological behavior. ${ }^{3,11,20}$ Each dog had previously been evaluated through medical history, physical examination, routine blood tests, thoracic radiographs and other appropriate imaging studies.

101 Information retrieved from all medical records included: signalment (age, sex, breed), concurrent diseases, presentation (primary versus recurrent), number of previous surgeries, involved sites, tumor size, histology, histological grade, clinical stage, treatment modality

104 (electron versus photon plan), response to radiation therapy, time to disease progression, 105 treatment-related complications, time and cause of death, and date of last follow-up visit. 106 Tumor site was classified as head (excluding oral and nasal cavity), trunk, and extremities. 107 Pathologic diagnoses were made by experienced pathologists (Dipl. ECVP) at the time of 108 treatment according to currently accepted criteria. ${ }^{21}$ Histological grade was based on a scale 109 of 3 (low, intermediate, high grade). ${ }^{3}$ The size of the tumor before radiation therapy was 110 evaluated by caliper measurement from physical examination, radiography, and/or computed 
111 tomography (CT), depending on anatomical site and clinicians' and/or owners' preferences.

112 Tumor volume was calculated by the rotational ellipse method (max. height $\mathrm{x}$ max. width $\mathrm{x}$

113 max. length $\mathrm{x} \pi / 6$ ). Staging workup for regional and distant disease included fine-needle

114 aspiration of the regional lymph node, thoracic radiography and abdominal ultrasound, and/or

115 total body CT, depending on clinicians' and/or owners' preferences. Stage was established

116 with the modified TNM staging system. ${ }^{22}$

Treatment

119 All dogs were treated with external beam megavoltage radiation therapy. Radiation was 120 delivered with a 6 megavolt (MV) linear accelerator (Dynaray LA20; ABB, Clinac DMX or 121 Clinac iX, Varian, Palo Alto, USA) using either photons (3-dimensional conformal radiation therapy, 3DCRT) or electrons, depending on tumor size and location. Treatment planning was performed on the basis of CT for photon plans or by hand calculation for electron plans. For treatment planning, the Eclipse External Beam Planning system version 8.1 or 10.0 (Varian

125 Oncology Systems, Palo Alto, CA) was used, applying the pencil beam convolution- (version 126 8.6.14) or AAA-algorithm (10.0.28). Dogs were under general anesthesia for planning-CT 127 and daily treatment, and - if treated with photon plans - immobilized in an individually 128 shaped vacuum cushion (BlueBag BodyFix, Elekta AB, Stockholm, Sweden). Dogs with 129 tumors in the head area were additionally immobilized with a custom-made bite block. ${ }^{23}$ The 130 GTV (gross tumor volume) was delineated using co-registered or in parallel viewed contrast131 enhanced CT images, and CTV (clinical target volume), accounting for subclinical 132 microscopic disease extension of $2-3 \mathrm{~cm}$ (presumed local infiltration) as well as positive 133 regional lymph nodes), was defined. The CTV-margin was then extended three-dimensionally 134 by $2 \mathrm{~mm}$ (for image-guided photon treatment), or $4-6 \mathrm{~mm}$ (for non-guided photon treatment) 
135 to define the planning target volume (PTV), accounting for internal physiologic movements,

136 patient motion, and setup uncertainties. Organs at risk were segmented as applicable.

137 The recommendations for specifying dose and volumes as proposed for veterinary

138 medicine were adhered to as proposed in the corresponding literature. ${ }^{24-26}$ For photon

139 plans, the prescribed dose was $30 \mathrm{~Gy}$ at the ICRU reference point ${ }^{25}$, delivered in 5

140 fractions of 6 Gy applied twice per week, resulting in an overall treatment time of 2.5

141 weeks. Treatment was performed isocentrically with bolus and wedges to ensure dose

142 homogeneity for photon treatments. For tumors treated with electron fields, the

143 according fields were applied at $100 \mathrm{~cm}$ source surface distance (SSD) and field sizes

144 as well as energies were chosen to adequately cover the tumor with the additional

145 suspected margin of infiltration of $2-3 \mathrm{~cm}$. The $90 \%$ isodose line was chosen to

146 encompass this target volume and for dose normalization. ${ }^{27}$

147 Radiation-related toxicity was graded according to the Veterinary Radiation Therapy

148 Oncology Group (VRTOG) scheme at each treatment, and 2-3 weeks after having

149 completed the protocol, thereafter monthly rechecks were scheduled. ${ }^{28}$ Specific

150 attention was paid to wound-healing complications, in-field fractures, vascular

151 complications, and second malignancies. The recommendation for use of metronomic

152 chemotherapy was based on the judgment of the clinicians managing the cases and on

153 owners' preferences, and consisted of oral administration of thalidomide (Thalidomide

154 CEG, Celgene srl, Milan, Italy; $1-2 \mathrm{mg} / \mathrm{kg} /$ per day), piroxicam (Feldene, Pfizer, Rome,

155 Italy; $0.3 \mathrm{mg} / \mathrm{kg} / \mathrm{per}$ day) and cyclophosphamide (Endoxan, Baxter, Rome, Italy; 7

$156 \mathrm{mg} / \mathrm{m}^{2} /$ every other day). All drugs were re-formulated and administered to the nearest

$1575 \mathrm{mg}$.

158

159

Outcome and follow-up 
160 Outcome information was obtained by means of medical records or communication with

161 referring veterinarians and owners at the end of the study period. Follow-up care included a

162 medical history and physical examination at progressive intervals beyond treatment. Response

163 data was noted according to the RECIST criteria for dogs. ${ }^{29}$ Complete remission was defined

164 as the disappearance of all target lesions. Partial response (PR) was defined as a reduction of

165 at least $30 \%$ in the sum of diameters of target lesions from baseline. Stable disease (SD) was

166 defined as $<30 \%$ decrease or $>20 \%$ increase in sum of diameters of target lesions from

167 smallest sum while on treatment. Progressive disease (PD) was defined as an increase in the

168 sum of diameters of target lesions by at least $20 \%$ over the size present at entry on study, or

169 the appearance of new lesions. Responses were required to last for at least 28 days. Follow-up

170 imaging was individualized based on baseline risk, symptoms, and clinicians' and/or owners'

171 preferences, and included thoracic radiographs and abdominal ultrasound or total body CT.

\section{Statistical analysis}

174 Statistical evaluation was performed together with a biostatistician (M.R.) and computed with 175 a commercial statistical software package (IBM ${ }^{\circledR}$ SPSS $^{\circledR}$ Statistics, Version 22). Description

176 of quantitative data characteristics, other than progression-free interval (PFI) and overall

177 survival $(\mathrm{OS})$, is given by mean $( \pm \mathrm{SD})$, unless otherwise specified. For qualitative

178 characteristics absolute and relative frequencies were provided. Association of discrete patient

179 or tumor specific variables on the addition of adjuvant metronomic chemotherapy was

180 evaluated with the Chi-square-test or logistic regression. For logistic regression odds ratio

181 (OR) together with the corresponding 95\% CI(OR) were computed.

182 PFI was defined as the interval between the first fraction of radiation therapy to measurable

183 progression of disease, while OS was defined as the interval between the first fraction of

184 radiation therapy and death. Dogs that were still free of progression at the time of data 
evaluation were censored. For OS, all deaths were considered events and dogs that were still

186 alive at the time of data evaluation or lost to follow-up were censored. The Kaplan-Meier survival analysis was followed by logrank or Breslow-Gehan-Wilcoxon tests. In case the survival curves did not cross the logrank test was applied. Otherwise the Breslow-GehanWilcoxon test was used. The multivariable Cox-regression analysis was used to determine whether the following factors were significantly associated with PFI or OS: presentation

191 (primary versus recurrent), number of previous surgeries, involved sites (head and neck versus trunk versus extremities), tumor size, histology, histological grade (1 versus 2 versus 3), regional lymph node metastasis (present versus absent), distant metastasis (present versus absent), medical treatment (yes versus no). Survival estimates are presented as median with the corresponding $95 \%$ confidence intervals $(95 \% \mathrm{CI})$. In case median survival was not reached, the mean survival with the corresponding 95\%CI was reported. For Cox-regression hazard ratio (HR) together with the corresponding $95 \% \mathrm{CI}(\mathrm{HR})$ were computed. For factors not only the global p-value but also the pairwise Bonferroni-corrected post-hoc tests were reported. A p-value of $<0.05$ was considered significant for all analyses. For between levels comparisons of three-level factors within Cox-regression the Bonferroni correction was applied to significance $0.05 / 3=0.016$ and tendency $0.1 / 3=0.03$ levels to perform the pairwise Bonferroni-corrected post-hoc tests.

\section{Results}

Patient and tumor characteristics

206 Fifty dogs fulfilled the inclusion criteria for this study, of which 21 were female (15 spayed) 207 and 29 were male (9 neutered). The dogs were of various pure ( $\mathrm{n}=36)$ and mixed breeds $208(n=14)$ and a total of 19 breeds were represented. The ages ranged from 4-16 years with a 209 mean of $9.8( \pm 2.7)$ years. The weight ranged from $8-52 \mathrm{~kg}$, with a mean of $29.3( \pm 10.4) \mathrm{kg}$. 
210 Of the $17(34 \%)$ dogs that had previous surgeries, $9(18 \%)$ had one and 8 dogs $(16 \%)$

211 had 2. Pretreatment tumor volumes ranged from 0.06 to $491 \mathrm{~cm}^{3}$, with a mean of 118.3

$212 \mathrm{~cm}^{3}( \pm 125)$. Six tumors $(12 \%)$ were located on the head, $5(10 \%)$ on the trunk, and the

213 remaining $39(78 \%)$ on the limbs. Of the 50 cases, 19 tumors $(38 \%)$ were

214 histologically described as perivascular wall tumors (including hemangiopericytoma,

215 spindle cell sarcoma, myopericytoma), 13 (26\%) as peripheral nerve sheet tumors, 8

$216(16 \%)$ as fibrosarcoma, $7(14 \%)$ as undifferentiated/anaplastic sarcoma, $2(4 \%)$ were

217 myxosarcomas, and $1(2 \%)$ was a liposarcoma. Histological grade was given in 42

218 cases $(84 \%)$ with $52 \%$ grade $1(n=26), 26 \%$ grade $2(n=13)$, and $6 \%$ grade 3 tumors

$219(\mathrm{n}=3)$. For these dogs the modified TNM staging was applied and revealed 40 tumors

220 to be of stage I, and 2 of stage II. For the remaining 8 cases, the histological samples

221 were not available and grade could not be retrieved. None of the dogs presented with

222 either regional or distant metastasis.

\section{Treatment protocol, side effects}

225 Thirty $(60 \%)$ dogs were treated with single electron fields with energies ranging from 12-15

$226 \mathrm{MeV}$, and 20 cases (40\%) had a 3D-conformal photon plan. Acute side effects were assessed 227 in 42 cases and consisted of grade 0 toxicity (33.3\%) in $14 \mathrm{dogs}$, grade 1 toxicity $(64.3 \%)$ in

22827 dogs (erythema, dry desquamation, alopecia or epilation) and grade 2 toxicity $(2.3 \%)$ in

229 one dog (patchy, moist desquamation, without edema). Late effects were assessed in 37 cases

230 with no toxicity (67.6\%) in 25 dogs, and grade 1 toxicity (32.4\%) in 12 dogs (leukotrichia).

231 Twenty dogs received additional metronomic chemotherapy. Neither the histological

232 grouping $(\mathrm{p}=0.067)$ nor tumor location $(\mathrm{p}=0.245)$ nor volume $(\mathrm{p}=0.232)$ was significantly

233 relevant for the adjunction of chemotherapy. However, the proportion of dogs receiving

234 metronomic chemotherapy decreased with increasing age. Elderly dogs received metronomic 
235 chemotherapy less often (OR: $0.713(95 \% \mathrm{CI}: 0.551-0.923), \mathrm{p}=0.01)$ than younger dogs, with a

$23629 \%$ of administration reduction for each additional year of age.

Response to treatment

239 Follow-up time for censored cases $(\mathrm{n}=17)$ was 561 days (95\%CI:482-640, range 87-929

240 days). Of the 33 animals that were known to have died, 15 (45.5\%) died of tumor-related 241 causes and $18(54.5 \%)$ died of tumor -unrelated causes. At the end of radiation therapy, all $242(100 \%)$ dogs were evaluated for response. There were $5(10 \%)$ complete responses, $10(20 \%)$ 243 partial responses, and $35(70 \%)$ stable diseases. At 3-month re-evaluation, 41 (92\%) dogs

244 were assessed for response, and there were 6 complete responses, 13 partial responses, 20 245 stable diseases, and 2 progressive diseases. At 6-mont re-evaluation, 32 (64\%) dogs were 246 assessed for response, and there were 8 complete responses, 7 partial responses, 13 stable 247 diseases, and 4 progressive diseases. Median progression free interval for all cases was 419 248 days (95\%CI:287-551). Tumor location significantly influenced PFI, with tumors on the 249 limbs (median PFI: 466 days (95\%CI:321-611)) faring significantly better than tumors on the 250 head or on the trunk (median PFI: 110 days (95\%CI:0-238) and 203 days (mean, median not 251 reached ( $\mathrm{SE}=31$ days $(95 \% \mathrm{CI}: 142-264)$, respectively) $(\mathrm{p}=0.021) ;)$; (head vs. trunk $(\mathrm{p}=0.421$, $252 \mathrm{~ns})$, head vs. $\operatorname{limb}(\mathrm{p}=0.004$, significant $)$, trunk vs. $\operatorname{limb}(\mathrm{p}=0.409, \mathrm{~ns})$ after Bonferroni 253 correction) (Figure 1).

254 The number of previous surgeries also significantly influenced PFI. Dogs having had $>1$ prior 255 surgery had the shortest PFI (105 days (95\%CI:0-349)), whereas dogs with no prior surgery 256 or with one prior surgery were free of progression for a median of 420 days $(95 \% \mathrm{CI}: 285-$ $257556)$ and 536 days ( $95 \% \mathrm{CI}: 92-980)(\mathrm{p}=0.003)$; (no surgery vs. one surgery $(\mathrm{p}=0.579$, ns), no 258 surgery vs. $>1$ surgeries ( $\mathrm{p}=0.02$, tendency), one surgery vs. $>1$ surgeries $(\mathrm{p}=0.027$, 259 tendency)), respectively. Histology $(\mathrm{p}=0.080)$, tumor grade $(\mathrm{p}=0.167)$, radiation therapy 
260 modality $(\mathrm{p}=0.585)$, age $(\mathrm{p}=0.296)$, and pretreatment volume $(\mathrm{p}=0.658)$ did not significantly 261 influence PFI.

262 Median overall survival was 513 days (95\%CI:368-658)-Regarding histology, a significantly

263 shorter survival in the fibrosarcoma group versus all other histologies was found (median 190

264 days $(95 \% \mathrm{CI}: 38-343))(\mathrm{p}<0.001)$; grade did not influence survival $(\mathrm{p}=0.922)$. However,

265 location was documented to be a significant prognostic factor: dogs with tumors on the limbs

266 survived longer (579 days (95\% CI:452-706) than those with tumors on the head (195 days

267 (95\%CI: 51-339)) and on the trunk (190 days (95\%CI: 57-323)), $(\mathrm{p}=0.031)$; (head vs. trunk

$268(\mathrm{p}=0.366, \mathrm{~ns})$, head vs. $\operatorname{limb}(\mathrm{p}=0.038, \mathrm{~ns})$, trunk vs. $\operatorname{limb}(\mathrm{p}=0.015$, significant $)$ after

269 Bonferroni correction) (Figure 2).

270 No association was found for tumor grade $(p=0.631)$, treatment modality $(p=0.735)$, or age

$271(\mathrm{p}=0.412)$, however, increasing tumor volume decreased OS (HR 1.004 (95\%CI:1.001-1.007),

$272 \mathrm{p}=0.005)$. The number of previous surgeries showed no significant association with OS.

273 The addition of metronomic chemotherapy did not influence the duration of PFI, however,

274 dogs receiving metronomic chemotherapy had a significantly longer OS (757 days

275 (95\%CI:570-944) compared with dogs that did not receive systemic treatment (286 days

276 (95\%CI:0-518), $(\mathrm{p}=0.023))$ (Figure 3). Interestingly, 3 dogs receiving metronomic

277 chemotherapy that achieved partial response $(n=2)$ or stable disease $(n=1)$ at the end of

278 radiation therapy obtained complete response at 6-month re-evaluation, and 1 dog that had

279 stable disease at the end of radiation therapy obtained partial response at 6-month re-

280 evaluation. 


\section{Discussion}

282 The present study is based on a large number of dogs with soft tissue sarcoma with macroscopic (unresected or recurrent) disease treated with a hypofractionated 5x6 Gy radiation therapy protocol. For several reasons, such as financial or logistical restraints of the owners, co-morbidities of the patients amongst others, there seems to be a demand for noncurative approach for the treatment of canine soft tissue sarcoma. In a previous study, a 4x8

287 Gy palliative protocol for macroscopic disease revealed short progression free interval of 5.2 months and overall survival of 10.3 months. ${ }^{11}$ The progression free interval and overall survival from the current study population were higher, with progression free interval of 13.9 months and overall survival of 17.1 months. Limb tumors fared significantly better with 291 progression free interval of 15.5 months regardless of tumor burden, histology or grade. The general finding of long progression free interval and overall survival was surprising, as a satisfactory outcome of radiation therapy alone for soft tissue sarcoma is not usually expected,

294 due to the rather low radioresponsiveness of sarcomas. Also, although the great majority of 295 dogs obtained stable disease at the end of radiation therapy, $10 \%$ and $20 \%$ of the treated cases 296 obtained complete responses and partial responses, respectively. Interestingly, the response 297 rate further improved over time, highlighting that neoplastic cells keep dying off for months 298 after the end of radiation treatment or respond slowly to metronomic chemotherapy.

299 The commonly used term "canine soft tissue sarcoma" is used for tumors with similar 300 biologic behavior. ${ }^{2,30}$ However, while not described for surgical or multimodal curative 301 approach $^{3-5}$, tumors on the limbs might respond better to hypofractionated protocols ${ }^{12}$, or the 302 limb location displays simply less discomfort for the patient. ${ }^{11}$ Furthermore, this result may be 303 attributable to the fact that the majority of limb soft tissue sarcomas are histologically 304 described as perivascular wall tumors. ${ }^{1}$ Compared to anaplastic sarcomas, it is known that 305 they usually have less expansile growth with less tissue infiltration, low to no local aggressive 
behavior with recurrence developing after a long latency, and low tendency to metastasize. ${ }^{1,31}$

308 Management of recurrent tumors is often more difficult, and this study showed that more than

309 one previous surgery in dogs presenting with macroscopic disease drastically reduced

310 progression free interval to 3.4 months, while overall survival was not influenced. Multiple

311 excisions may disrupt or contaminate the tissue planes, cause damage to existing vasculature

312 with possible subsequent tissue hypoxia and could hypothetically lead to a selection pressure

313 of cells with a higher malignancy in the recurrent tumor. Furthermore, recurrence of

314 incompletely excised soft tissue sarcomas has been linked to histological grade ${ }^{32}$, with a low

315 recurrence rate to be expected in a microscopic disease setting of mostly low-grade soft tissue

316 sarcomas. $^{12}$ The results of the current study also support this finding.

317 Larger tumor size is in general considered a poor predictive factor ${ }^{2,6}$, however, while most

318 likely influencing the probability for complete surgical excision, size itself has not been

319 identified as a prognostic factor in various studies. ${ }^{30,32,33}$ In this study, increasing tumor

320 volume decreased overall survival, perhaps indicating that larger tumors cause greater

321 discomfort and prompt owners into the decision of euthanasia earlier.

323 Metronomic chemotherapy refers to the frequent administration of cytotoxic drugs at doses

324 significantly less than the maximum tolerated dose, with no prolonged drug-free breaks,

325 leading to an anti-angiogenic effect and immune-modulation. ${ }^{34}$ Unlike traditional

326 chemotherapy, the main targets of which are proliferating tumor cells, the main targets of

327 metronomic chemotherapy are the endothelial cells of the growing vasculature of a tumor. ${ }^{34}$

328 Although the use of dose-intense chemotherapy has been controversial in dogs with soft tissue

329 sarcomas, the addition of metronomic chemotherapy has been recently described in the

330 microscopic setting, leading to significantly prolonged disease-free intervals compared to a 
331 nonrandomized control group. ${ }^{14}$ In the current study, dogs receiving metronomic

332 chemotherapy had a significant improvement in overall survival, whereas the progression free

333 interval was not increased. Although encouraging, this finding needs to be interpreted

334 cautiously. First, it should be borne in mind that OS may not be the best endpoint in

335 veterinary oncology, as it may be influenced by tumor-unrelated factors, such as owners'

336 financial concern or logistic issues, leading to premature euthanasia. Second, dogs receiving

337 metronomic chemotherapy were younger than those that only received radiation therapy, and

338 it may be possible that owners of younger dogs were more motivated in treating their animals

339 than owners of elderly patients, which may have elected euthanasia sooner. Several reasons

340 may explain why elderly dogs are less likely to receive chemotherapy, including the presence

341 of concomitant diseases and the owner' unwillingness to accept possible negative effects of

342 systemic treatment.

343 Interestingly, some dogs receiving metronomic chemotherapy experienced an improved

344 response rate over time, with 3 cases obtaining complete response after partial response $(\mathrm{n}=2)$

345 or stable disease $(n=1)$, and 1 case obtaining partial response after having achieved stable

346 disease at the end of radiation therapy. Whether the addition of metronomic chemotherapy

347 after radiation therapy is superior to radiation therapy alone needs to be tested in prospective

348 trials. With this premise in mind, this study indicates that metronomic chemotherapy warrants

349 further prospective investigation in canine soft tissue sarcoma to answer several questions.

350 Indeed, the results obtained here did not allow identifying a subgroup of dogs that are more

351 likely to benefit from metronomic chemotherapy.

352

353 Acute toxicity of the radiation protocol (mostly grade $0-1$ skin reactions) was completely

354 healed within two to three weeks after completion of treatment. During the time of follow-up, 355 there was no evidence of late effects, which - due to the lower total dose and smaller fraction 
356 size - are also expected to a lower degree than in the prior described $4 \times 8$ to 9 Gy protocols. ${ }^{11,}$

$357{ }^{12}$ Compared to Lawrence et al, where the „biologic equivalent dose“ (BED) ${ }^{35}$ was calculated

358 to be $117.3 \mathrm{~Gy}_{3}$ (for late effects) ${ }^{11}$ the comparable BED in this study is lower with $90 \mathrm{~Gy}_{3}$.

359 For the future, it could be considered to increase the dose per fraction of this protocol slightly,

360 in order to improve response rate with a likely acceptable complication rate.

361 Also, toxicity of metronomic chemotherapy was mild as appropriate for a palliative approach,

362 and allowed outpatient treatment. These findings are in accordance with previous studies. ${ }^{11,12,}$

365 Due to the retrospective nature of this study, the use of archival data, and considering the

366 well-known difficulty of proper clinical assessment of response of soft tissue sarcoma to

367 radiation therapy, the authors may not have been able to describe the full range of

368 improvement in these dogs and some of the information had to be gathered by telephone

369 follow-up, possibly skewing the information by owner's perception. Furthermore, different

370 pathologists made the diagnoses and histological grade was only available in $84 \%$ of the

371 cases. The modified stage grouping could only be applied in patients with known grade. ${ }^{22}$ As

372 most of the patients were of stage I disease and the prognostic significance of the staging

373 system for soft tissue sarcoma has not been further investigated ${ }^{2}$, the TNM was excluded from

374 statistical description. The bi-institutionality of the study is of minor concern, as the treatment

375 planning was either done remotely (all photon plans) or under the direct supervision (electron

376 hand calculations) by the same radiation oncologist (CRB).

378 The standard of care for soft tissue sarcoma remains aggressive local therapy, which often

379 involves a combination of surgery and full-course post-operative radiation therapy. With this

380 treatment, median times to recurrence have been described with 700 to 798 days ${ }^{4,5}$ and were 
381 hence considerably longer than the progression free interval of 419 days obtained with the

382 much more simple protocol described herein. However, for dogs with non-resectable tumors,

383 the herein described hypofractionated 5x6 Gy protocol is well tolerated and yields a

384 satisfactory clinical outcome with negligible toxicity found in the clinically relevant observed

385 time-frame. As with all hypofractionated protocols, a small risk of additional late toxicity

386 with longer observation remains. Future prospective studies are warranted to determine

387 whether metronomic chemotherapy after radiation therapy is beneficial for dogs with

388 macroscopic soft tissue sarcoma. 


\section{References}

390 1. Avallone G, Boracchi P, Stefanello D, Ferrari R, Rebughini A, Roccabianca P. Canine 391 perivascular wall tumors: high prognostic impact of site, depth, and completeness of margins. 392 Vet Pathol 2013;51:713-721.

393 2. Liptak JM, Forrest L. Soft Tissue Sarcomas. In: Withrow SJ, Vail D, Page RL (eds):

394 Withrow and MacEvens's Small Animal Clinical Oncology. St. Louis, Missouri: Elsevier 395 Saunders, 2013;356-380.

396 3. Kuntz CA, Dernell WS, Powers BE, Devitt C, Straw RC, Withrow SJ. Prognostic 397 factors for surgical treatment of soft-tissue sarcomas in dogs: 75 cases (1986-1996). J Am Vet 398 Med Assoc 1997;211:1147-1151.

399 4. McKnight JA, Mauldin GN, McEntee MC, Meleo KA, Patnaik AK. Radiation 400 treatment for incompletely resected soft-tissue sarcomas in dogs. J Am Vet Med Assoc $401 \quad 2000 ; 217: 205-210$.

402 5. Forrest LJ, Chun R, Adams WM, Cooley AJ, Vail DM. Postoperative radiotherapy for 403 canine soft tissue sarcoma. J Vet Intern Med 2000;14:578-582.

404 6. Ehrhart N. Soft-tissue sarcomas in dogs: a review. J Am Anim Hosp Assoc $405 \quad 2005 ; 41: 241-246$.

406 7. Scarpa F, Sabattini S, Marconato L, Capitani O, Morini M, Bettini G. Use of 407 histologic margin evaluation to predict recurrence of cutaneous malignant tumors in dogs and 408 cats after surgical excision. J Am Vet Med Assoc 2012;240:1181-1187.

409 8. Chase D, Bray J, Ide A, Polton G. Outcome following removal of canine spindle cell 410 tumours in first opinion practice: 104 cases. J Small Anim Pract 2009;50:568-574.

411 9. Bateman KE, Catton PA, Pennock PW, Kruth SA. 0-7-21 radiation therapy for the 412 palliation of advanced cancer in dogs. J Vet Intern Med 1994;8:394-399. 
413 10. Thrall DE, LaRue SM. Palliative radiation therapy. Semin Vet Med Surg (Small 414 Animals), 1995;205-208.

415 11. Lawrence J, Forrest L, Adams W, Vail D, Thamm D. Four-fraction radiation therapy 416 for macroscopic soft tissue sarcomas in 16 dogs. J Am Anim Hosp Assoc 2008;44:100-108.

417 12. Demetriou JL, Brearley MJ, Constantino-Casas F, Addington C, Dobson J. Intentional 418 marginal excision of canine limb soft tissue sarcomas followed by radiotherapy. J Small Anim 419 Pract 2012;53:174-181.

420 13. Kung MB, Poirier VJ, Dennis MM, Vail DM, Straw RC. Hypofractionated radiation 421 therapy for the treatment of microscopic canine soft tissue sarcoma. Vet Comp Oncol 2014.

422 14. Elmslie RE, Glawe P, Dow SW. Metronomic therapy with cyclophosphamide and 423 piroxicam effectively delays tumor recurrence in dogs with incompletely resected soft tissue 424 sarcomas. J Vet Intern Med 2008;22:1373-1379.

425 15. Burton JH, Mitchell L, Thamm DH, Dow SW, Biller BJ. Low-dose cyclophosphamide 426 selectively decreases regulatory T cells and inhibits angiogenesis in dogs with soft tissue 427 sarcoma. J Vet Intern Med 2011;25:920-926.

428 16. Buchholz J, Hagen R, Leo C, Ebling A, Roos M, Kaser-Hotz B, et al. 3D conformal 429 radiation therapy for palliative treatment of canine nasal tumors. Vet Radiol Ultrasound $430 \quad 2009 ; 50: 679-683$.

431 17. Cancedda S, Rohrer Bley C, Aresu L, Dacasto M, Leone VF, Pizzoni S, et al. Efficacy 432 and side effects of radiation therapy in comparison with radiation therapy and temozolomide 433 in the treatment of measurable canine malignant melanoma. Vet Comp Oncol 2014.

434 18. Fidel J, Schiller I, Hauser B, Jausi Y, Rohrer-Bley C, Roos M, et al. Histiocytic 435 sarcomas in flat-coated retrievers: a summary of 37 cases (November 1998-March 2005). Vet 436 Comp Oncol 2006;4:63-74. 
19. Poirier VJ, Bley CR, Roos M, Kaser-Hotz B. Efficacy of radiation therapy for the

438 treatment of macroscopic canine oral soft tissue sarcoma. In Vivo 2006;20:415-419.

439 20. Ciekot PA, Powers BE, Withrow SJ, Straw RC, Ogilvie GK, LaRue SM.

440 Histologically low-grade, yet biologically high-grade, fibrosarcomas of the mandible and 441 maxilla in dogs: 25 cases (1982-1991). J Am Vet Med Assoc 1994;204:610-615.

442 21. Hendrick MJ, Mahaffey AE, Moore FM, al. e. Histological Classification of the

443 Mesenchymal Tumors of Skin and Soft Tissues of Domestic Animals. In: Pathology

444 Pathology DC: Armed Forces Institute of Pathology American Registry of Pathology (ed):

445 World Health Organization, International Histologic Classification of Tumors of Domestic 446 Animals. Washington, 1998.

447 22. Liptak JM, Forrest L. Soft Tissue Sarcomas. In: Withrow SJ, Vail D (eds): Withrow 448 and MacEvens's Small Animal Clinical Oncology. St. Louis, Missouri: Elsevier Saunders, $449 \quad 2007 ; 425-454$.

450 23. Rohrer Bley C, Blattmann H, Roos M, Sumova A, Kaser-Hotz B. Assessment of a 451 radiotherapy patient immobilization device using single plane port radiographs and a remote 452 computed tomography scanner. Vet Radiol Ultrasound 2003;44:470-475.

453 24. International Commission of Radiation Units and Measurements. Prescribing, 454 recording, and reporting photon beam therapy, ICRU Report 50. Bethesta, MD: ICRU, 1993.

455 25. International Commission of Radiation Units and Measurements. Prescribing, 456 recording, and reporting photon beam therapy (Supplement to ICRU Report 50), ICRU

457 Report 62. Bethesda, MD: ICRU, 1999.

458 26. Keyerleber MA, McEntee MC, Farrelly J, Podgorsak M. Completeness of reporting of 459 radiation therapy planning, dose, and delivery in veterinary radiation oncology manuscripts 460 from 2005 to 2010. Vet Radiol Ultrasound 2012;53:221-230. 
461 27. Das IJ, Cheng CW, Healey GA. Optimum field size and choice of isodose lines in

462 electron beam treatment. Int J Radiat Oncol Biol Phys 1995;31:157-163.

463 28. Ladue T, Klein MK. Toxicity criteria of the veterinary radiation therapy oncology

464 group. Vet Radiol Ultrasound 2001;42:475-476.

465 29. Nguyen SM, Thamm DH, Vail DM, London CA. Response evaluation criteria for 466 solid tumours in dogs (v1.0): a Veterinary Cooperative Oncology Group (VCOG) consensus 467 document. Vet Comp Oncol 2013.

468 30. Dennis MM, McSporran KD, Bacon NJ, Schulman FY, Foster RA, Powers BE.

469 Prognostic factors for cutaneous and subcutaneous soft tissue sarcomas in dogs. Vet Pathol $470 \quad 2011 ; 48: 73-84$.

471 31. Stefanello D, Morello E, Roccabianca P, Iussich S, Nassuato C, Martano M, et al.

472 Marginal excision of low-grade spindle cell sarcoma of canine extremities: 35 dogs (1996473 2006). Vet Surg 2008;37:461-465.

474 32. McSporran KD. Histologic grade predicts recurrence for marginally excised canine 475 subcutaneous soft tissue sarcomas. Vet Pathol 2009;46:928-933.

476 33. Bostock DE, Dye MT. Prognosis after surgical excision of canine fibrous connective 477 tissue sarcomas. Vet Pathol 1980;17:581-588.

478 34. Mutsaers AJ. Metronomic chemotherapy. Top Companion Anim Med 2009;24:137479143

480 35. Hall EJ. Time, dose, and fractionation in radiotherapy. Radiobiology for the 481 Radiologist. Philadelphia: Lippincott Williams \& Wilkins, 2000;397-417.

$483 \quad$ Figure captions

484 Figure 1: Progression-free interval for all cases grouped by tumor location (dotted line: limbs, 485 solid line: trunk, dashed line: head). 
486 Figure 2: Overall survival times for all cases grouped by tumor location (dotted line: limbs, 487 solid line: trunk, dashed line: head).

488 Figure 3: Overall survival times for patients treated without (dashed line) or with (solid line) 489 metronomic chemotherapy. 


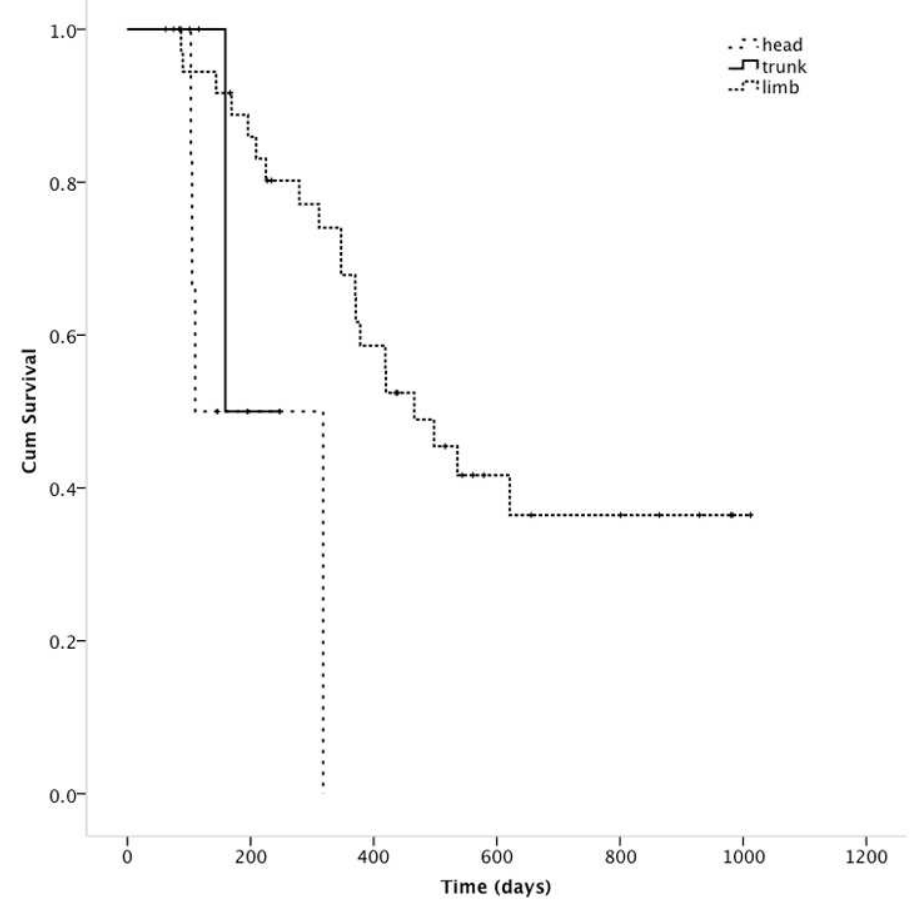




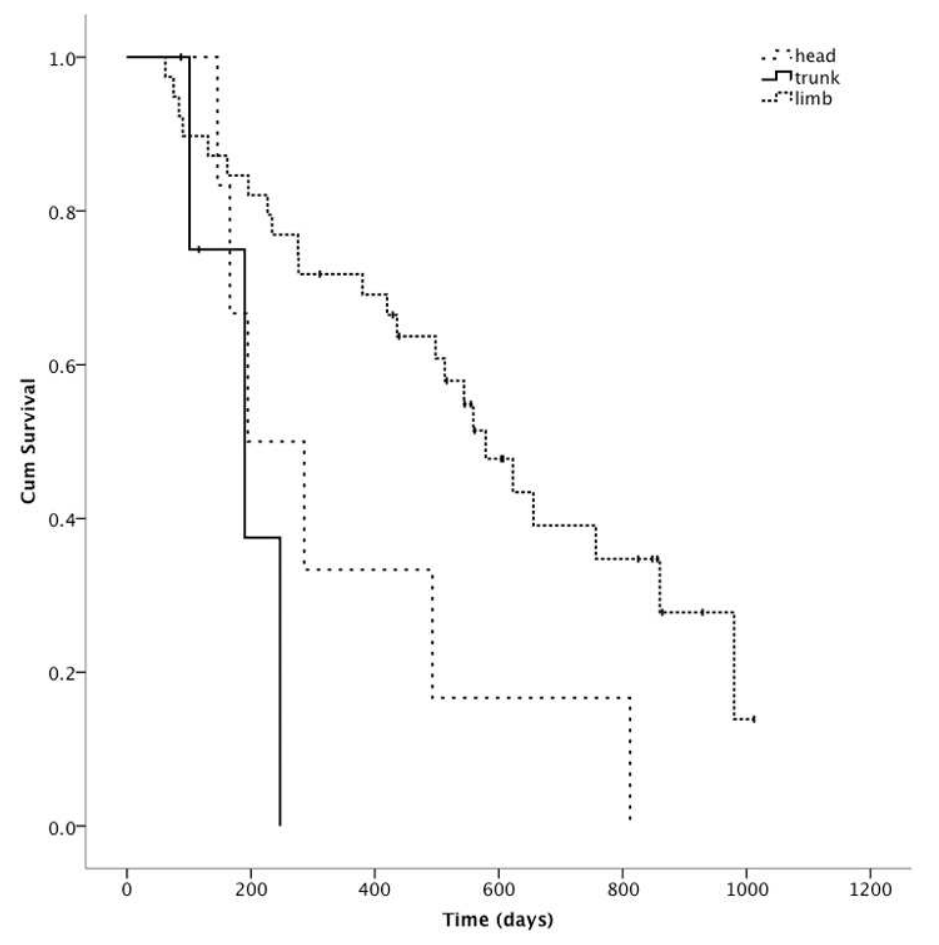




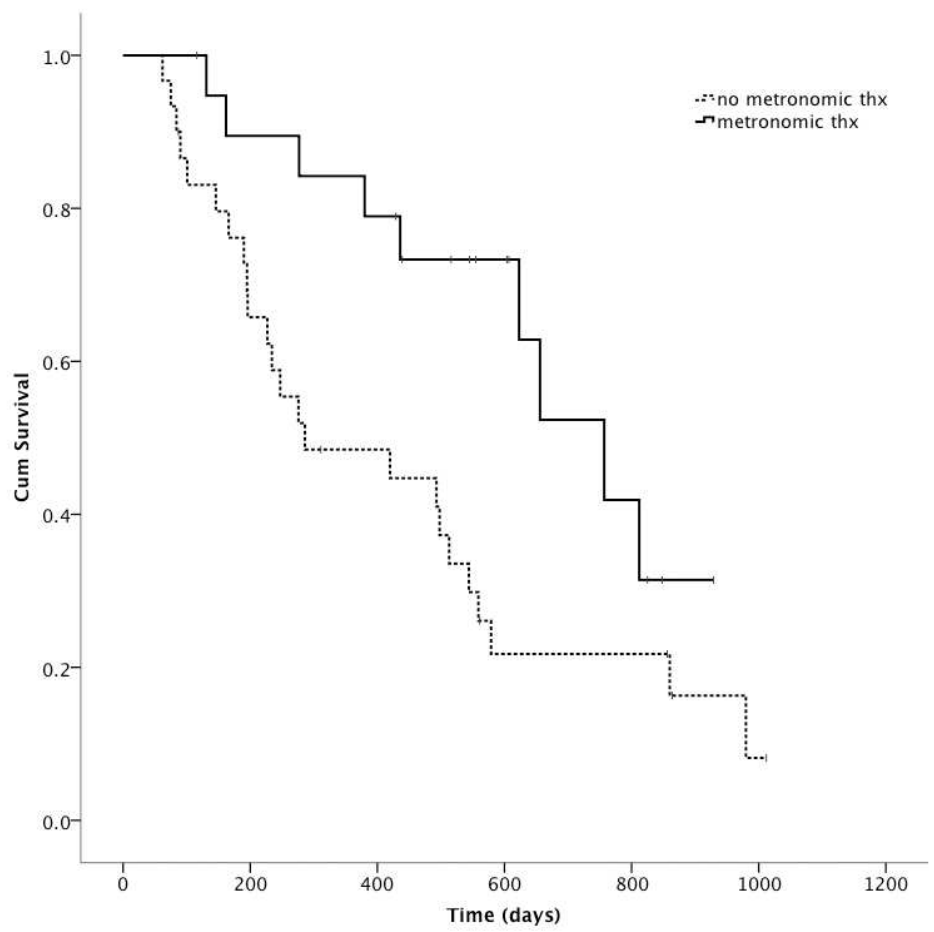

